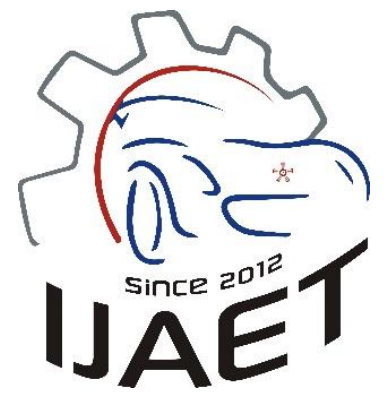

e-ISSN: 2146 - 9067

International Journal of Automotive

Engineering and Technologies

journal homepage:

https://dergipark.org.tr/en/pub/ijaet

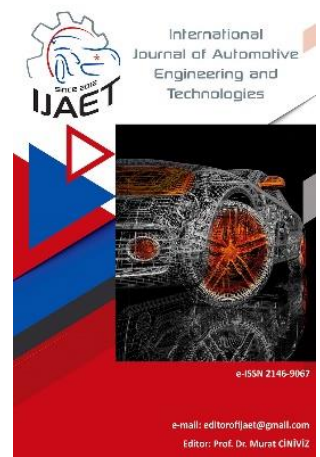

Original Research Article

\title{
Investigation of effects of surface roughness on the performance of cam mechanisms
}

Hasan Baş ${ }^{1, *}$

1, * Department of Mechanical Engineering, Faculty of Engineering, Karadeniz Technical University, Trabzon, Turkey

\author{
ARTICLE INFO \\ 1. 0000-0002-5653-3813 \\ Doi: $10.18245 /$ ijaet.843229 \\ * Corresponding author \\ bas@ktu.edu.tr \\ Received: Dec 19, 2020 \\ Accepted: Feb 27, 2021 \\ Published: Mar 31, 2021 \\ Published by Editorial Board \\ Members of IJAET \\ (C) This article is distributed by \\ Turk Journal Park System under \\ the CC 4.0 terms and conditions.
} \begin{abstract}
The surface roughness of two contacting surfaces significantly influences the tribological performance of the mechanical elements. Their impression is more pronounced under the mixed elasto-hydrodynamic lubrication condition. The cam and flat follower mechanism is a typical sample in which adverse tribological conditions, including direct boundary interactions occurs. In this study, effects of surface roughness on the friction force and friction coefficient are investigated using engine oil at different test conditions in a cam follower mechanism. It is seen that decreasing roughness of the contact surfaces has a more desirable tribological performance, and decrease friction coefficient, therefore increase wear resistance.
\end{abstract}

Keywords: Surface Roughness, Oil Film, Friction Coefficient, Combustion Engine Oil

\section{Introduction}

The effect of surface roughness pattern on the tribological performance of mechanical elements is an important issue that has attracted much attention for more than four decades. The properties of surface layers are crucial to understand the tribological performance. Various machine components such as bearings, gearbox, camshaft, crank shaft, etc. require excellent tribological properties and practices to ensure longer life. Understanding engineered surfaces is very important for solving many scientific problems that involve friction, contact mechanics, heat conduction, electric current conduction, and component design [1]. Surface roughness has been the subject of experimental and theoretical investigation for a long time. Surface texture is one of the most important parameters that controls the tribological behavior. Patir and Cheng developed a stochastic analysis approach to study the effects of surface roughness height and distribution between components upon the lubrication of machine elements, [2], [3]. The approach has also been used to assess cam and follower performance e.g. Refs. [4], [5]. For the direct acting cam and follower situation shown in Fig. 1, Dowson et al. [3] studied the predicted effects of surface roughness upon nominal film thickness, power loss and load carried by the asperities. Due to the fact that there are still very few examples of attempting to reduce friction in boundary lubrication conditions with high contact pressure such as cam/follower combination, the effects of surface texturing remain unknown. Ehret et al. [6] have studied 
the effects of various surface textures, described by either an orientated waviness or a uniform distribution of asperities, for rolling and sliding conditions. Surface texture can lead to important differences and in pure sliding it is shown that the best lubrication condition is produced for transverse waviness. The definitive linking of surface texture to improved machine element performance in, say, a cam and follower, would be a powerful technique [5]. O'Connor and Spedding [7] undertook an experimental study of the effect of surface waviness on a cam profile. Such waviness was influenced by the form imposed onto the grinding wheel by the roller dresser. Wear and oil retention characteristics of the automobile camshaft surface were investigated. It was suggested that the wavy cam surface could offer good oil retention characteristics but that with time the release of hard wear particles due to the wave peaks could be problematical. It was proposed that a cam surface having the same roughness, but zero or reduced waviness, would show improved performance. Waviness on automotive cams remains an issue and it is interesting to note that analysts are now in a position to contribute to the debate on the overall influence [5], [8]. For tribologists, the most critical interface in the valve train is the cam and follower due to the problem of providing effective lubrication. Often, it was assumed that the cam and follower operated in the boundary lubrication regime; however, more recent investigations have shown that mixed and elastohydrodynamic lubrications are the dominant regime.

The cam and follower lubrication is one of the interesting issues of transient EHDlubrication that has rarely been investigated specially for rough surfaces. Thus, effects of surface roughness on the friction force and friction coefficient are investigated. The results can provide guidance on the tribological application of cam and follower in industrial application.

\section{Theoretical Bases}

\subsection{Force analysis}

In the cam mechanism, the contact load acting on the cam-follower contact area varies depending on the cam rotation angle. Maximum contact load occurs at cam angles where the cam nose is in contact with the follower.
The loading state of the roughness flat-surface follower-cam pair is shown in Figure 1. Here, the contact load is in the same direction as the centerline during a cam period due to the flatness of the follower surface. The contact load at the contact surface is constant only along the base circle. The cam surface acts on the follower surface with the cam pressure angle depending on the cam rotation angle. The maximum value of the pressure angle in question occurs in the cam position where the contact point of the two surfaces is furthest from the center line, as shown in Fig.1. Thus, the cam remains under the influence of the maximum torque at the rotation angles corresponding to these positions.

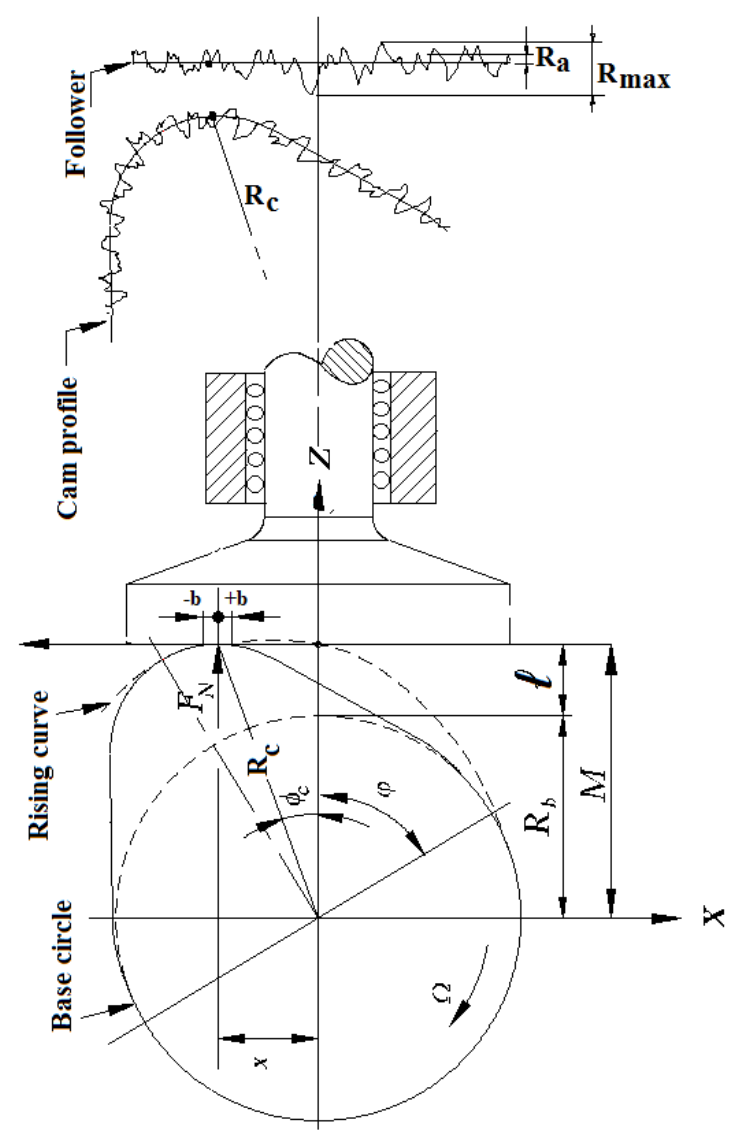

Figure 1. Roughness considerations in a cam and flat surfaced follower pair.

\subsection{Friction force}

The general form of the Reynolds equation for EHD lubrication of two smooth surfaces has been presented in many publications, such as [9]. Generally, when two surfaces contact each other in the presence of lubricant, pressure in the entire domain is governed by:

$$
\begin{aligned}
& \frac{\partial}{\partial x}\left(\frac{\rho h^{3}}{\eta} \frac{\partial p}{\partial x}\right)+\frac{\partial}{\partial y}\left(\frac{\rho h^{3}}{\eta} \frac{\partial p}{\partial y}\right)=6 u \frac{\partial}{\partial x}(\rho h)+ \\
& 12 \frac{\partial \rho h}{\partial t}
\end{aligned}
$$


where $\mathrm{p}$ is the hydrodynamic pressure, $\rho$ is the lubricant's density, $u$ is mean velocity of the surfaces, and $\eta$ is the viscosity of the lubricant. Film thickness, h, is defined as the gap between two surfaces. Therefore, when the surfaces are rough, the film thickness for the line contact problem can be expressed as following:

$h(x, y)=h_{0}+v(x, y)+\delta(x, y)$

Where, $h_{0}$ is minimum film thickness, $v$ and $\delta$ are the surface elastic deformation and the asperities height, respectively. The regime of true mixed lubrication, where there are both elâstohydrodynamic and boundary lubricated regions within a single contact, remains quite poorly understood. The cam-follower pair is a very complex lubricated contact because its continuous variation of load, speed and radius of curvature. In order to determine the friction force arising in the elasto-hydrodynamic contact under the forces acting through a period in the cam mechanism, instead of $h$ mentioned above, using which is the film thickness at the lubricated maximum hertz contact between cam and follower, the final calculation process can be write as follows.

$$
F_{s}=\frac{\eta_{0}\left(u_{1}-u_{2}\right)}{h_{c e n}} \cdot \ell \cdot \int_{-b}^{b} e^{\alpha \cdot p_{\max }} \sqrt{\left(1-\frac{x^{2}}{b^{2}}\right)} d x
$$

\section{Experimental Setup}

\subsection{Description of the test rig}

The test apparatus was manufactured in the Mechanical Engineering Department at Karadeniz Technical University. Detailed information about the test apparatus can be obtained from Ref. [10, 11, and 12]. The mechanical system and measurement unit of the test apparatus are designed to measure the frictional behavior between cam and follower at different loads, speeds and lubricating conditions. A lubricating system is designed to investigate the effect of different type of oils on the friction behavior at the cam / follower interface. In order to ensure safe and accurate data collection and storage, a computer and a data acquisition system are attached to the measurement unit and a computer program is written to evaluate this data.

\subsection{Measurement procedures}

In the test apparatus, two types of accelerometers were used to measure the mass force in vertical and horizontal directions due to acceleration of moving masses. The mass force in the horizontal direction is measured by using a piezoelectric type of accelerometer sensor, which works by a compression effect and is mounted rigidly between two linear bearings in the loading system. To determine the friction force and friction coefficient, detailed information about friction force applied to the follower by cam, causes a tensile effect on the measurement beam and the effect produces a signal proportional to the beam force, which can be used in the calculation of the friction force [12]. The measurements were recorded continuously, and friction force was recorded with the load cell. Temperature of samples was measured with a thermocouple connected to the follower. Tribological properties were determined at ambient temperature, in $70 \%$ relative humidity $(\mathrm{RH})$. The results were evaluated taking into consideration the forming a tribofilm in mixed or EHD lubrication.

In order to understand the effects of surfaces roughness on the tribological behavior in the cam-follower mechanism, surface roughness and morphologies of the contact surfaces and wear surfaces were investigated using a surface roughness test apparatus and scanning electron microscopy. In this study, roughness of the surfaces of cam and followers are measured using contact profilometers. The most common method of studying surface texture features is the contact-type stylus profilometer. A fine, very lightly loaded stylus is dragged smoothly at a constant speed across the surface under examination. The height position of the stylus generates an analog signal which is converted into a digital signal that is stored, analyzed, and displayed, and the output provides a magnified view of the original profile.

A photograph of 2D contact-type profilometer and the profile of mean roughness ( $\mathrm{Ra})$, roughness depth $(\mathrm{Rz})$ and maximum roughness (Rmax) formulations are shown in Fig. 2.

Figure 3 Shows, roughness measurements of the worn surfaces of cam and follower before and after lubricated and non-lubricated tests were performed using a surface roughness tester (Mahr-Germany Perthen Perthometer) pursuant to DIN 4768, as a measure of (Ra), (Rz) and (Rmax). 


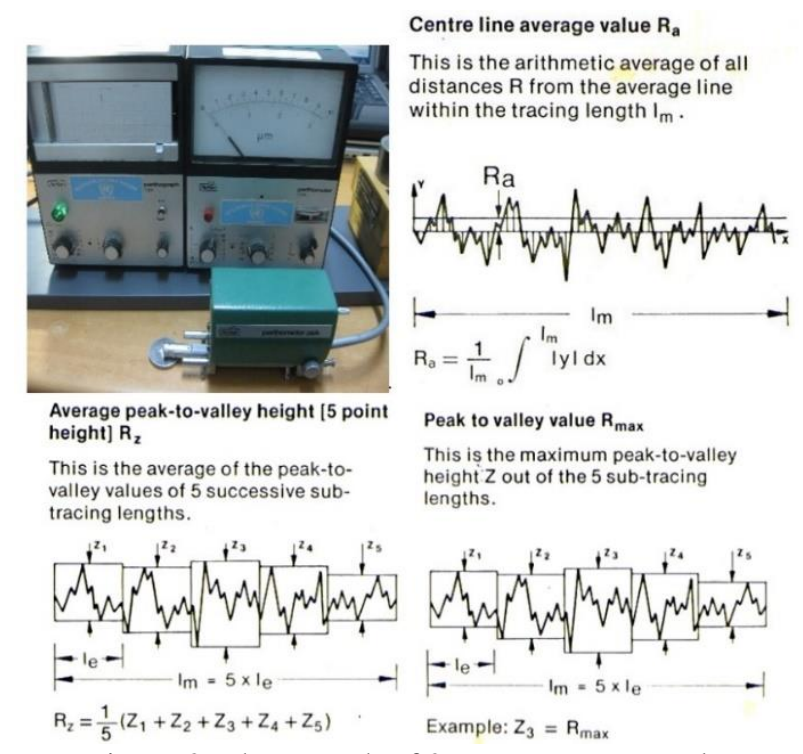

Figure 2. Photograph of 2D contact-type stylus profilometer and roughness formulations of $\mathrm{Ra}, \mathrm{Rz}$ and $\mathrm{Rmax}$

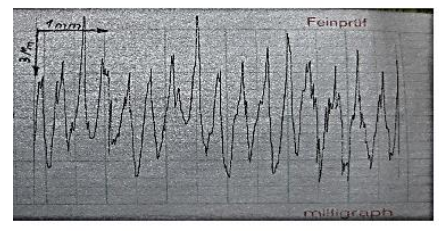

(a)

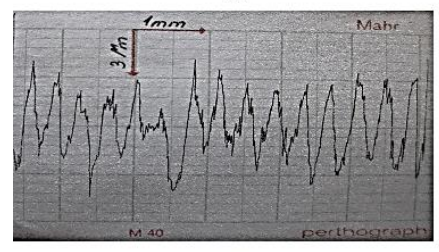

(c)

(e)

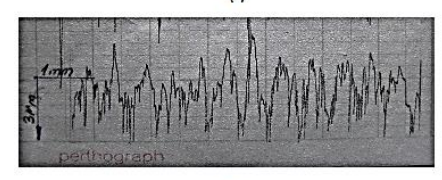

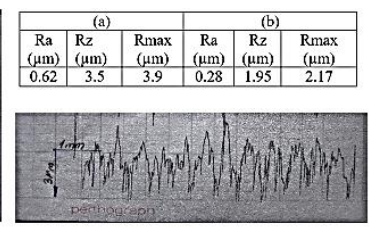

(b)
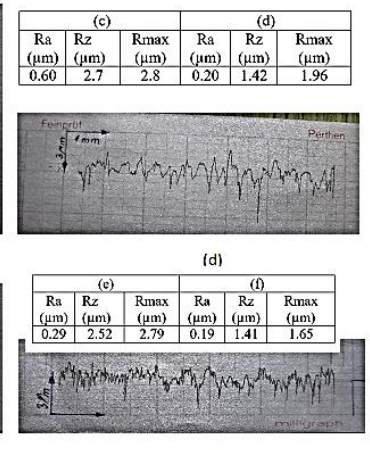

(f)
Figure 3. Graphical representation of the measured roughness profile of the cam and followers, (a) before,

(b) after non-lubricated (dry) tests of follower1; (c) before, (d) after lubricated tests of follower 3; (e) before, (f) after lubricated test of the cam.

It is seen that the result of surface roughness of the cam and follower tests with non-lubricated conditions are higher than lubricated conditions.

\subsection{Test methodology}

In this study, to investigate tribological behavior of surface roughness on the cam mechanism, a new automotive cam shaft and four followers used. Followers has a different roughness manufactured by grinding from the same material. Chemical composition of the cam and follower were given in Table 1 . In the manufactured test apparatus, cam is direct acting to the follower. Followers with different surface roughness initially run against nominally same cam for at a fixed camshaft speed and temperature. Experiments were conducted at $600 \mathrm{rpm}$, and $312 \mathrm{~N}$ contact load. Experimental periods were selected as 10 minutes for every test after running-in, and the average values of at least three tests were taken.

Table 1. Chemical composition of the cam and follower.

\begin{tabular}{lcc}
\hline & Cam & Follower \\
\hline $\mathrm{C}$ & 3.42 & 1.06 \\
$\mathrm{Si}$ & 2.15 & 0.3 \\
$\mathrm{Mn}$ & 0.6 & 0.3 \\
$\mathrm{P}$ & 0.05 & 0.007 \\
$\mathrm{~S}$ & 0.01 & 0.01 \\
$\mathrm{Cr}$ & 0.9 & 1.48 \\
$\mathrm{Ni}$ & 0.95 & 0.13 \\
$\mathrm{Mo}$ & 0.05 & 0.04 \\
$\mathrm{Cu}$ & 0.10 & 0.23 \\
$\mathrm{Al}$ & 0.01 & 0.013 \\
$\mathrm{Ti}$ & 0.004 & 0.003 \\
\hline
\end{tabular}

\section{Results and Discussion}

\subsection{Tribological behavior}

Fig. 4 shows normal force, friction force and friction coefficient in a cam period lubricated by base oil (20W50 engine oil) and non-lubricated i.e. dry condition at $312 \mathrm{~N}, 600 \mathrm{rpm}, 40^{\circ} \mathrm{C}$. It is seen that, in case of lubricated and dry condition, the maximum contact load increases along with the cam profile and reaches its maximum value at cam nose, (i.e. at cam angles around $90^{\circ}$ ). Both of lubricated and nonlubricated situation, the value of contact load around the cam nose has same value at the same preload. However, the fric tion force and thus the friction coefficient are different. It is seen that the friction values in the oil-free contact are greater than that lubricated contact.

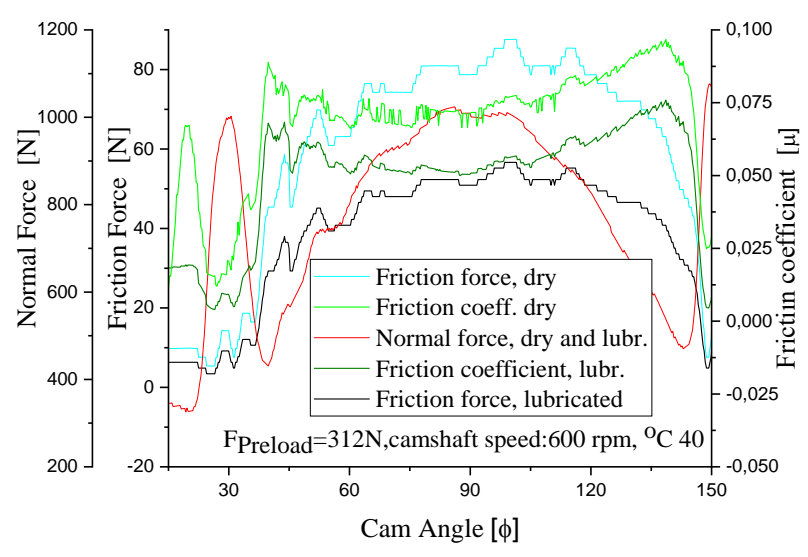

Figure 4: Normal force, friction force and friction coefficient in a cam period lubricated with the base oil (20W50 engine oil) at $312 \mathrm{~N}, 600 \mathrm{rpm}$ 
In order to investigate the tribological behavior of surface roughness in dry and lubricated contact, two followers were used with very close surface roughness $\mathrm{Ra}$ value to each other. Figure 5 illustrates the variation of the surface roughness with the number of cycles when used follower1 and follower3 under dry and lubricated conditions. Both of roughness values of the followers are decreases with increasing cycle number of the cam. It is seen that the slope in the curves approaches zero after 3000 turns. Based on these results, it can be stated that the oily contact has a rather abrasion effect and is subject to abrasion, whereas in dry contact, the adhesion effect is more intense due to scuffing on the surface. In the running-in period, the asperities are gradually flattened and friction coefficient is progressively reduced until reaching the steady state.

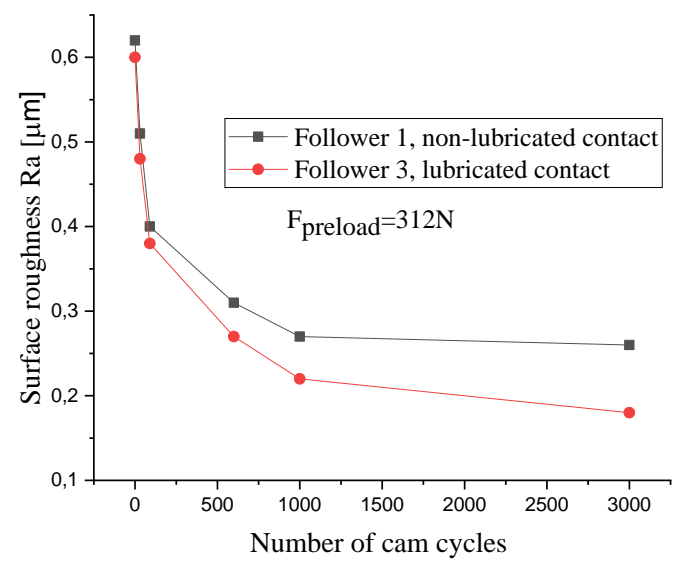

Figure 5. Variation of friction coefficient with number of cycle using follower 1 and follower 3 under dry and lubricated conditions.

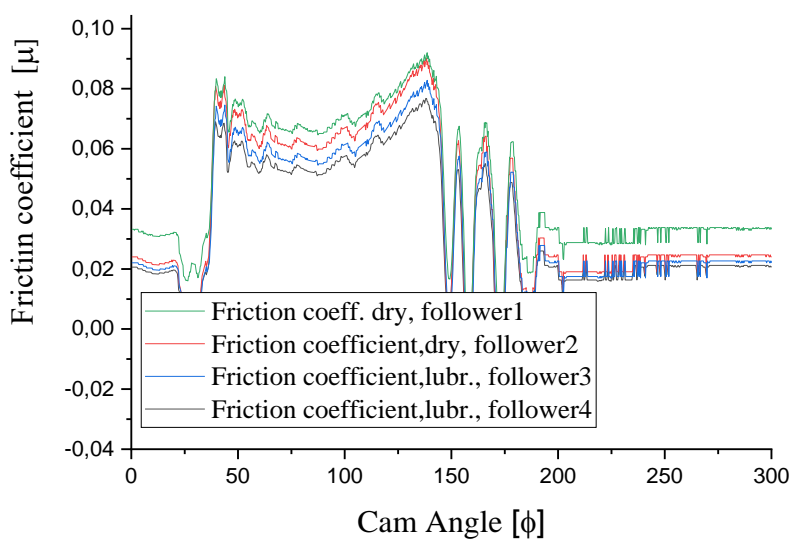

Figure 6. Variation of friction coefficient with cam angle when used follower1-4 under dry and lubricated conditions.

It is important to note that the surface texture of four followers (different surface roughness values) after running-in plays an important role in controlling the frictional behavior. Figure 6 illustrates the variation of coefficient of friction with surface roughness under dry and lubricated conditions. The friction coefficient as a function of contact roughness to be able to diagnose the effect of roughness on the lubricated and nonlubricated condition were done. Surface roughness of follower1 and follower3 are given in Fig. 3 above. $\mathrm{Ra}, \mathrm{Rz}$ and $\mathrm{Rmax}$ values of follower2 and 4 after running-in, are $0.16[\mu \mathrm{m}]$, $1.18[\mu \mathrm{m}], 1.22[\mu \mathrm{m}]$ and $0.13[\mu \mathrm{m}], 0.52[\mu \mathrm{m}]$, $0.58[\mu \mathrm{m}]$, respectively. As shown in Fig. 6 , the friction coefficient of the follower1 is slightly higher than that of the follower2 under the nonlubricated condition.

In addition, the friction coefficient values of the follower3 is quite higher than that of follower4 under the lubricated condition. All friction coefficient curves showed the same trend. It was observed that the percentage of reduction in friction coefficient under dry conditions were higher than that of lubricated condition. Hence, it is understood that in dry contact, the adhesion effect is more intense due to scuffing on the cam-follower surfaces.

To identify the effect of roughness on lubricated and non-lubricated state, frictional force tests were made as a function of contact roughness. Figure 7 shows the variation of friction force with surface roughness under bot of dry and lubricated conditions. As shown in Fig. 7, the friction coefficion of the follower1 is slightly higher than that of the follower2 under the nonlubricated condition.

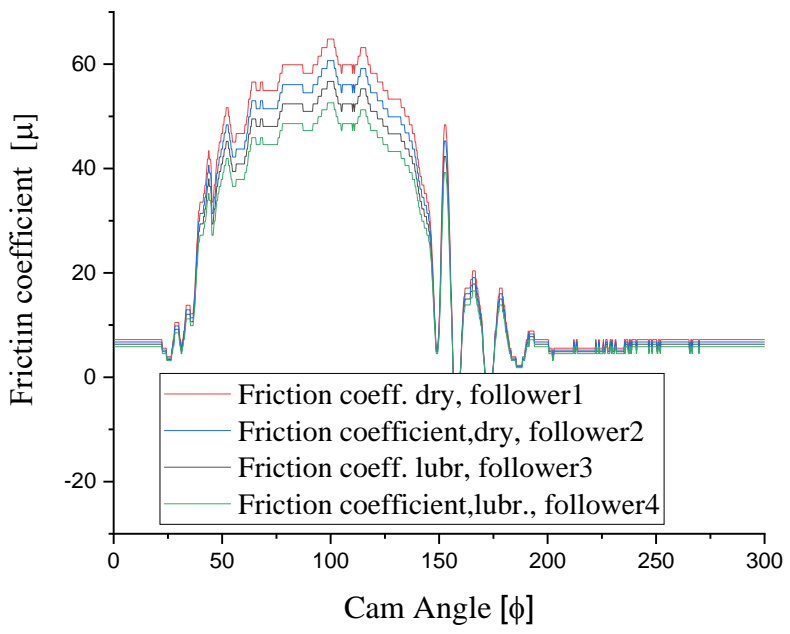

Figure 7. Variation of friction force with cam angle when used follower3 and follower4 under dry and lubricated conditions.

And also, the friction coefficion values of the 
follower3 is quiet higher than that of follower4 under the lubricated condition. All friction coefficient curves showed the same trend. It was observed that the percentage of reduction in friction coefficient under dry conditions were higher than that of lubricated condition.

According to the above results, it is clear that the surface roughness indeed has an important role on of friction values during sliding. Thus, surface textures were characterized by means of surface roughness parameters.

Results displayed in Figure 6 and Figure 7 shows that for rising values of roughness is increasing, friction coefficient and friction force increases too. It can be explained that high roughness leads to smaller contact areas leading directly to high local contact pressures on the contrary to profiles with a low Ra.

Figures $8 \mathrm{a}-8 \mathrm{c}$ shows scanning electron microscope morphologies of the contact surfaces before test, and Figures $8 \mathrm{~d}-8 \mathrm{f}$ after dry test under 312 preloads at $600 \mathrm{rpm}$ and ambient temperature.

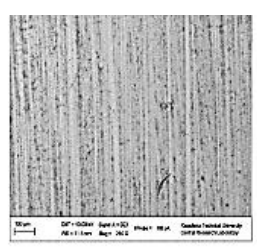

(a)
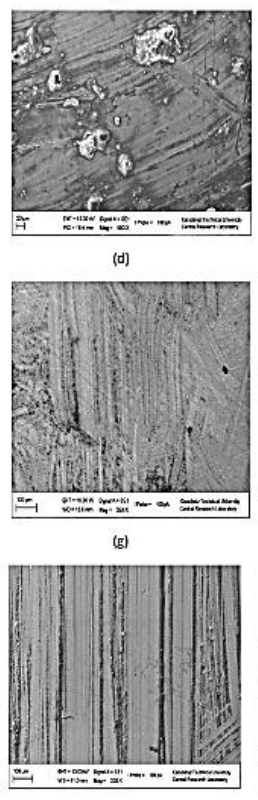

(u)
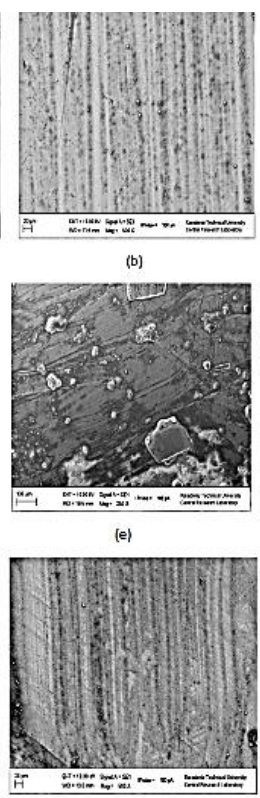

(h)

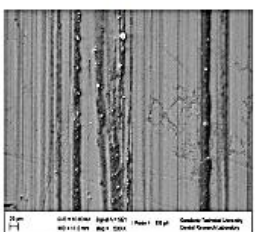

(k)

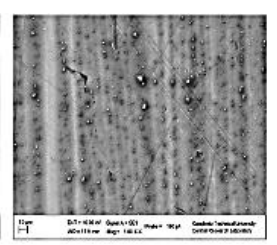

(c)
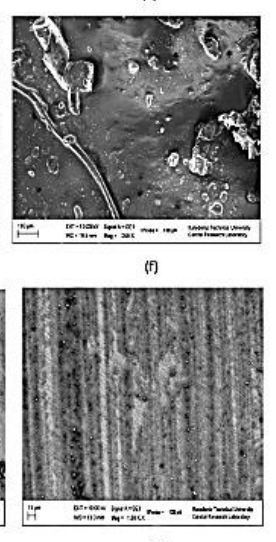

(i)

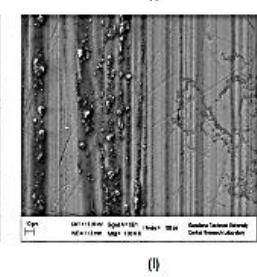

Figure 8. SEM morphologies of contact surfaces; nonlubricated i.e. dry contact tests (a)-(c) before test and (d)(f) after test; lubricated contact tests (g)-(i) before test and (j)-(1) after test under 312 preload, at $600 \mathrm{rpm}$. In this chapter, attempts have been made to study various surface roughness values.
As seen in Figs. 8d-8f, the worn surface of the follower lubricated by base oil was characterized by slight adhesion wear. For lubricated conditions, Figs. 8g-8i and Figs. 8j81 shows morphologies of the contact surfaces before and after the tests, respectively. As seen in Figs. 8j-81, the worn surface of the follower was characterized by abrasion wear, some adhesion wear.

\section{Conclusion}

Contact loads of the cam and follower were 312 $\mathrm{N}$. These were very near Hertzian pressure becomes in EHL, and contact surface nonconformal. Therefore, the system may be evaluated in a mixed lubrication regime, and local micro EHL zones may form at the interface of roughness surfaces. From the above experimental results, following conclusions can be drawn:

- the surface roughness has an important role on of friction values in engine parts during sliding, especially on the cam and follower,

- $\quad$ in dry contact, the adhesion effect is more intense due to scuffing on the camfollower surfaces.

- In the running-in period, the asperities are gradually flattened and friction coefficient is progressively reduced until reaching the steady state.

- as the roughness values increase, the friction coefficient and friction force also increase.

\section{Acknowledgment}

This research was funded by the Karadeniz Technical University, Scientific Research Projects Unit, (Project Code: 2008.112.003.9.

\section{References}

1. A. Torabi, S. Akbarzadeh, M. R. Salimpour and M. Taei, "Effect of surface roughness pattern on transient mixed elastohydrodynamic lubrication" Surface Topography: Metrology and Properties, Volume 4, Number 1, 2015

2. N. Patir, H.S. Cheng, "An average flow model for determining effects of threedimensional roughness in partial hydrodynamic lubrication" J. Lubr. Technol., Trans. ASME 100 $1197812-17$. 
3. D. Dowson, C.M. Taylor, G. Zhu, Mixed lubrication of a cam and flat faced follower, Proc. 13th Leeds-Lyon Symposium on Tribology - Fluid Film Lubrication, Tribol. Ser. vol. 11 Elsevier, Amsterdam, 1987, pp. 599-609.

4. L.S. Yang, A. Ibo, H. Negishi, A valve train friction and lubrication analysis model and its application in a camrtappet wear study, SAE Technical Paper 962030, 1996, 9 pp.

5. [5] M. Priest, C.M. Taylor "Automobile engine tribology — approaching the surface" Wear 2412000 193-203.

6. P. Ehret, D. Dowson, C.M. Taylor, Timedependent solutions with waviness and asperities in EHL point contacts, Proc. 23rd Leeds- Lyon Symposium on Tribology Elastohydrodynamics '96, Tribol. Ser. vol. 32 Elsevier, Amsterdam, 1997, pp. 313-324.

7. R.F. O'Connor, T.A. Spedding, Investigation into the effects of waviness present on the cam surface profile of an engine camshaft, Precis. Eng. 1121989 83-88, Butterworth.

8. I. Tanimoto, M. Kano, M. Sasaki, Establishment of a method for predicting cam follower wear in the material development process, SAE Technical Paper 902087, 1990, 8 pp.

9. Zhu D and Wang Q J 2013 Effect of roughness orientation on the elastohydrodynamic lubrication film thickness ASME J. Tribol. 135031501

10. H. Bas, Ph.D. Thesis, "Investigation of Friction Behavior of Additive Engine Oils on Cam Mechanisms," (in Turkish) Karadeniz Technical University Institute of Natural and Applied Sciences, 2001.

11. H. Bas, A. Biyıklıglu, "Investigation of Frictional Behavior of Different Kinds of Engine Oils on Cam Mechanisms Used in Automotive Engines," International Conference on Lubrication Techniques, Istanbul, 87-103, ICOLT'99.

12. H. Bas, A. Biyıklıglu and H. Cuvalci, "A New Test Apparatus For the Tribological Behavior Of Cam Mechanisms," Experimental Techniques, 2003. 